\title{
Retrospective Cross-Evaluation of an Histological and Deformable 3D Atlas of the Basal Ganglia on Series of Parkinsonian Patients Treated by Deep Brain Stimulation
}

\author{
Eric Bardinet ${ }^{1}$, Didier Dormont ${ }^{1,2}$, Grégoire Malandain ${ }^{3}$, \\ Manik Bhattacharjee ${ }^{5}$, Bernard Pidoux ${ }^{6}$, Christian Saleh $^{2}$, Philippe Cornu $^{7}$, \\ Nicholas Ayache ${ }^{3}$, Yves Agid ${ }^{4,5}$, and Jérôme Yelnik ${ }^{5}$ \\ ${ }^{1}$ CNRS UPR640-LENA, Hôpital Pitié-Salpêtrière, Paris, France \\ Eric.Bardinet@chups.jussieu.fr \\ ${ }^{2}$ Department of Neuroradiology, Hôpital Pitié-Salpêtrière, Paris, France \\ ${ }^{3}$ Epidaure group, INRIA, Sophia-Antipolis, France \\ ${ }^{4}$ Centre d'Investigation Clinique, Hôpital Pitié-Salpêtrière, Paris, France \\ ${ }^{5}$ INSERM U679, Hôpital Pitié-Salpêtrière, Paris, France \\ ${ }^{6}$ Neurophysiology Dpt, Hôpital Pitié-Salpêtrière, Paris, France \\ ${ }^{7}$ Department of Neurosurgery, Hôpital Pitié-Salpêtrière, Paris, France
}

\begin{abstract}
In functional neurosurgery, there is a growing need for accurate localization of the functional targets. Since deep brain stimulation (DBS) of the Vim thalamic nucleus has been proposed for the treatment of Parkinson's disease, the target has evolved toward the globus pallidus and subthalamic nucleus (STN) and the therapeutic indications have enlarged to include psychiatric disorders such as Tourette syndrome or obsessive compulsive disorders. In these pathologies, the target has been restrained to smaller functional subterritories of the basal ganglia, requiring more refined techniques to localize smaller and smaller brain regions, often invisible in routine clinical MRI. Different strategies have been developed to identify such deep brain targets. Direct methods can identify structures in the MRI itself, but only the larger ones. Indirect methods are based on the use of anatomical atlases. The present strategy comprised a 3D histological atlas and the MRI of the same brain specimen, and deformation methodology developped to fit the atlas toward the brain of any given patient. In this paper, this method is evaluated in the aim of being applied to further studies of anatomo-clinical correlation. The accuracy of the method is first discussed, followed by the study of short series of Parkinsonian patients treated by DBS, allowing to compare the deformed atlas with various per- and post-operative data.
\end{abstract}

\section{Introduction}

Since Deep Brain Stimulation (DBS) has been proposed for the first time for the treatment of tremor [1, the precise localization of the target has proved to be a crucial condition for the success of this therapeutic approach. Nowadays, this 
technique is used for the treatment of Parkinsonian patients, the subthalamic nucleus (STN) being the optimal target 2, that provides significative clinical benefit to the patient. The STN is a very small biconvex lens-shaped central grey nucleus oriented obliquely with reference to the three anatomical planes. In addition to playing a role in the pathophysiology of Parkinson's disease, the STN is also considered as being involved in the development of non motor diseases such as obsessive compulsive disorders [3]. In such pathologies, it is believed that a non motor portion of the STN could receive associative and limbic information from the associative and limbic portions of the external globus pallidus. It is thus more and more important to being able to localize with a high degree of precision not even the STN and the other nuclei of the basal ganglia, but also the topography of their functional subdivision into sensorimotor, associative and limbic territories.

With the progressive development of magnetic resonance (MR) imaging techniques, MR based methods have been developed for targeting the STN in individual patients [4] but the resolution of the sequence used (T2-weighted) is not precise enough and electrophysiological and clinical per-operative testing still remain necessary. Also, contrast in an MR image is related to relaxation times, a property which does not reflect systematically the histological structure of the nervous tissue. Different strategies have been developed to identify deep brain targets. Direct methods can identify structures in the MRI itself, but only the larger ones. Indirect methods are based on the use of anatomical atlases. To obtain a detailed cartography of the basal ganglia of a given patient, the only possible strategy is to adapt an histological atlas to the patient MR image. Various histological atlases have been developed in the past. With printed atlases [5]6 7], atlas-MR adaptation is to be done mentally by the atlas user. In [8], the authors recently proposed a method in which a digitized version of the Schaltenbrand \& Wahren (SW) atlas [7] is linearly coregistered interactively with the patient MR image. A similar technique 9] was previously applied to an atlas of the thalamus [6].

One motivation of the present work was to propose a tool allowing a better anatomical identification of the STN target. Our strategy was twofold. On the one hand, a three-dimensional (3D) anatomical atlas based on both histological and MR data of a postmortem brain specimen was constructed. Histological data were used to draw accurate contours of basal ganglia structures and functional territories. MR data were used to generate anatomically relevant 3D surfaces of these different structures by means of data fusion and contour optimization. On the other hand, image processing techniques were developed with which atlas surfaces could be deformed on an automatic basis onto any given patient's MR acquisition and could be sliced along any orientation. The most similar to the present study is [10] but it relied on the SW atlas, the contours of which are spatially inhomogeneous. In other methods there was no anatomical atlas but data acquisition through information learning 11121314.

In this article, basal ganglia histological mapping of Parkinsonian patients treated by DBS, achieved by automatic atlas deformation, is compared with 
various per- and post-operative data. Precisely, cross-evaluation is performed with per-operative electrophysiological recordings and with anatomical localization of therapeutic contacts obtained with an already published and clinically validated method [8].

\section{Material and Methods}

\subsection{Atlas Construction}

A human brain obtained at autopsy from body donation was submitted to T1and T2-weighted MRI sequences 36 hours after death. After brain extraction, the left hemisphere was fixed in formalin solution for 24 hours, cut into 1.5cm-thick frontal blocks that were fixed for 8 days and cut into 70-micron-thick frontal sections on a freezing microtome. Photographs of the frozen blocks were taken every ten sections. The 800 sections obtained were collected serially. One series of sections (every tenth section) was Nissl-stained. Another adjacent series was immunostained for calbindin. Contours of cerebral regions of the basal ganglia (striatum, globus pallidus, substantia nigra, subthalamic nucleus) and of their functional subterritories (sensorimotor, associative, limbic) and some related structures (thalamus, bed nucleus, pedunculopontine nucleus) were delimited on the basis of histological and immunohistochemical staining. Photographs of the frozen sections were aligned by using fiducial markers to obtain a geometrically consistent 3D cryo-block which was registered with the T1-MR and T2-MR sequences. Each histological section was registered onto the corresponding cryosection, compensating for histological processing distortions, thus providing 3D histo-blocks. All registrations were performed by applying the same automatic intensity-based method to a region of interest centered on the basal ganglia. Tracing of the contours was optimized by confronting all co-registered atlas data. 3D consistency of atlas structures was also optimized at this stage. Such contours were considered as the best estimate of the actual 3D geometry of the atlas brain. Surfaces were generated from these serial contours, yielding a true 3D atlas of the basal ganglia which could be sliced in any orientation (e.g. an AC-PC based plane or an oblique surgical trajectory) [15].

\subsection{Atlas Deformation}

The histological 3D atlas was adapted onto the brain of a given patient by automatic registration of the patient's T1-weighted MR image with the atlas post-mortem T1-weighted MR image, following a hierarchical framework. First, a global registration was computed, consisting in a rigid transform completed by an isotropic scaling factor. On the atlas MR image, an Region of Interest wasq extracted once and for all, embbeding the basal ganglia of the left hemisphere (dorso-ventral limits: corpus callosum, pons; medio-lateral limits: mid-sagittal plane, Sylvian fissure; antero-posterior limits: corpus callosum). This ROI was automatically extracted on the globally registered patient MR image, both for the left and right hemispheres. Then non-rigid (affine) registration was performed 
between atlas ROI and patient ROIs, yielding two transformations which were used to map the atlas independantly on the two hemispheres of the patient's brain. All registrations were performed by an iconic robust multiscale block matching algorithm using correlation as similarity measure.

\subsection{Evaluation}

Qualitative evaluation of the deformation was first performed by constructing average normalized images. Then, evaluation was performed on two retrospective series of data related to Parkinsonian patients treated by DBS. The first series (six patients) consisted in pre-operative T1-weighted (3D IR-FSPGR) MR images, used for targeting in stereotactic conditions, and in per-operative microelectrode recordings interpreted by an electrophysiologist. The second series (ten patients) consisted in post-operative T1-weighted MR images and in clinical outcome obtained by stimulation of the definitive therapeutic contacts (each electrode comprises four contacts which can be tested and selected post-operatively on the basis of their therapeutic efficacy).

\section{Results}

\subsection{Atlas Construction}

Fig. 1 summarizes the different steps of the atlas construction.
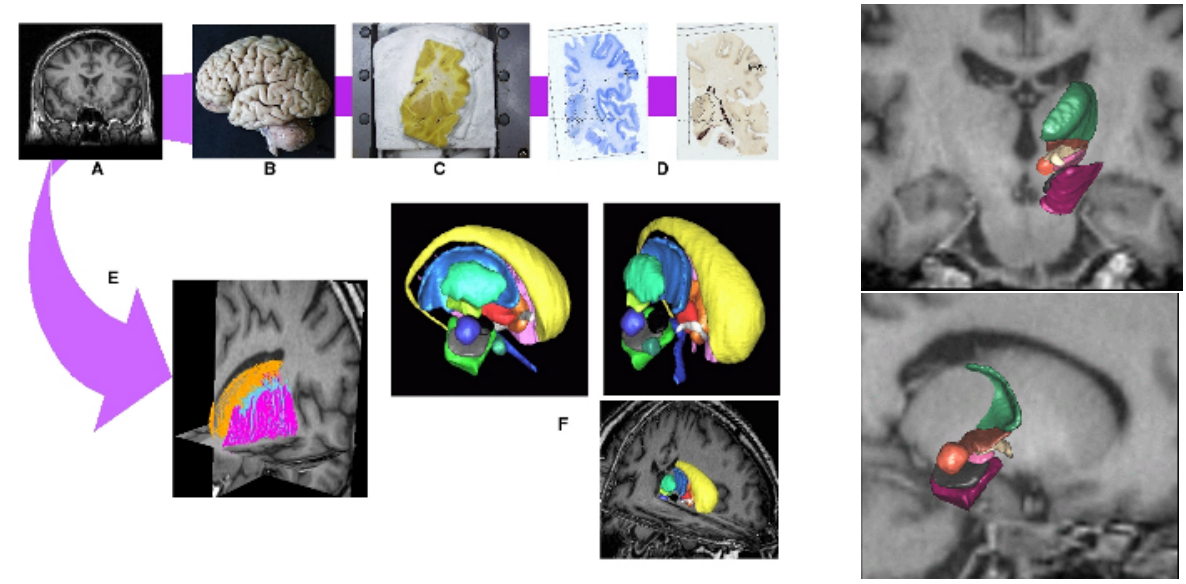

Fig. 1. Summary of atlas construction. Left block: A) MR (T1 and T2-weighted) acquisitions of the post-mortem specimen; B) brain extraction; C) frozen brain sectioning; D) histological colorations and contour tracing; E) coregistration of atlas data (cryo and histo 2D sections, MR images); F) resulting 3D meshes of the basal ganglia, after multimodal and 3D optimization. Right block: cerebral peduncle, substancia nigra, red nucleus, subthalamic nucleus, Forel field II, zona incerta and perithalamus with atlas T1-weighted MR image. 

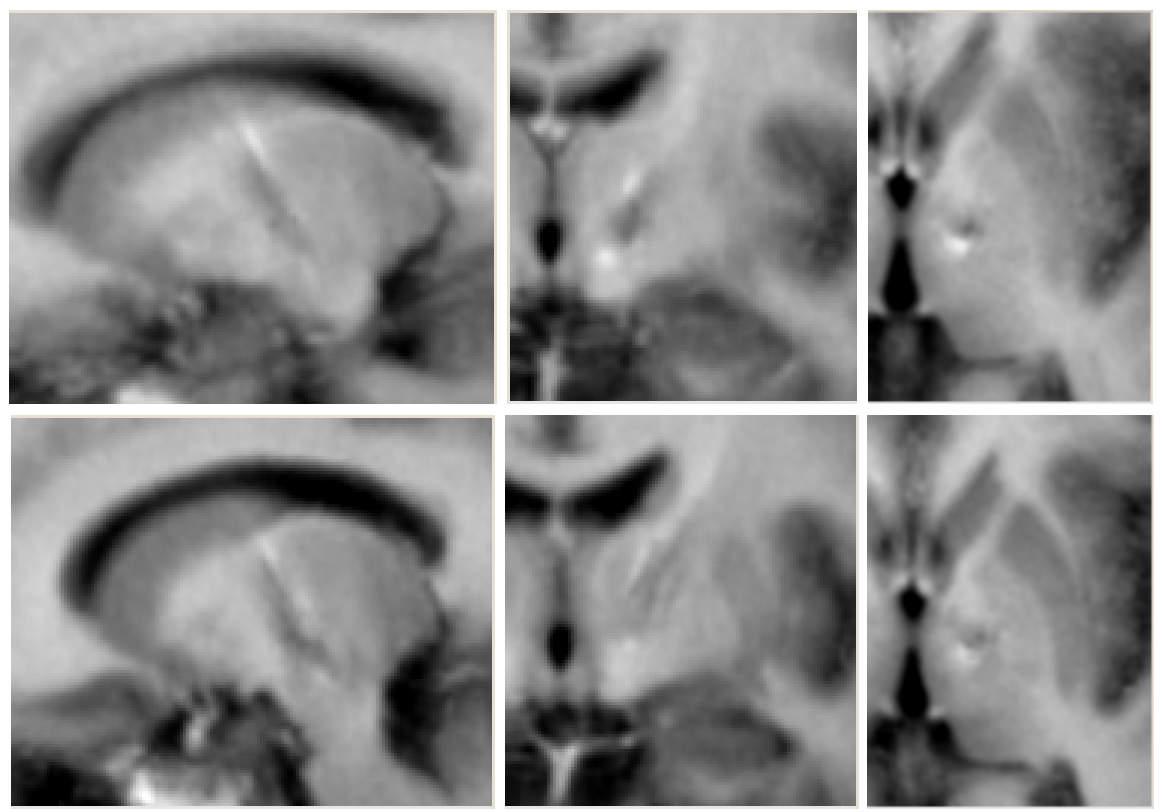

Fig. 2. Intensity normalized patient MR images averaged after ACPC (first row) and atlas spatial normalization (second row). Note that contours of the basal ganglia visible in MRI (caudate nucleus, putamen, thalamus) are sharper with the atlas normalization (only one hemisphere is presented, as the atlas is deformed indenpendantly on the 2 hemispheres).

\subsection{Evaluation}

Average Images. The 16 T1-weighted MR images of the patients included in this paper were normalized in intensity, spatially normalized and averaged. Spatial normalization was conducted following two distinct strategies (see Fig. 2). On the one hand, ACPC normalization was performed (the images were automatically aligned along their mid-sagittal planes, and scaling factors were applied along the antero-posterior direction to scale each image onto the AC$\mathrm{PC}$ line of the first patient). On the other hand, atlas normalization was performed (using the transformation computed during atlas deformation - see section 2.2).

\section{Comparison of Atlas-Based STN Contours Versus STN Revealed by} Per-operative Electrophysiological Recordings. Before the operation, the target and trajectory angles were chosen using MRI imaging [4. Electrophysiological activity was recorded during the operation using sets of two to four micro-electrodes, mounted on a microdrive attached to the stereotactic frame. Recordings were analyzed by the electrophysiologist every $0.5 \mathrm{~mm}$, from 5.5 $\mathrm{mm}$ above the target to $3 \mathrm{~mm}$ deeper. For each point, the intensity of the signal and the structure identified by the electrophysiologist were noted. To 

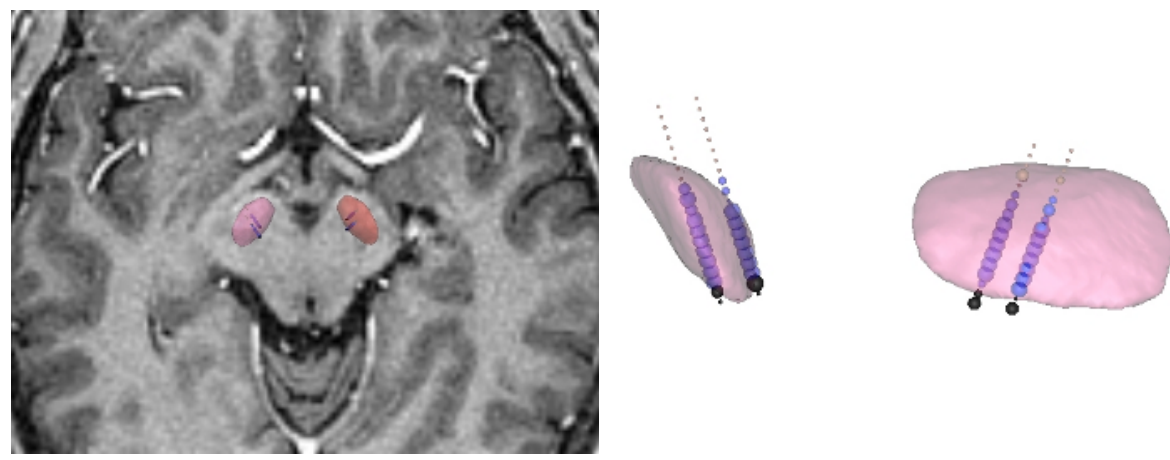

Fig. 3. Coregistration of the atlas structures and electrophysiological per-operative recordings in the patient's pre-operative MRI. The blue spheres represent the recordings of two microelectrodes by hemisphere identified as being within the STN by the electrophysiologist. Interval distance between two successive spheres is $0.5 \mathrm{~mm}$.
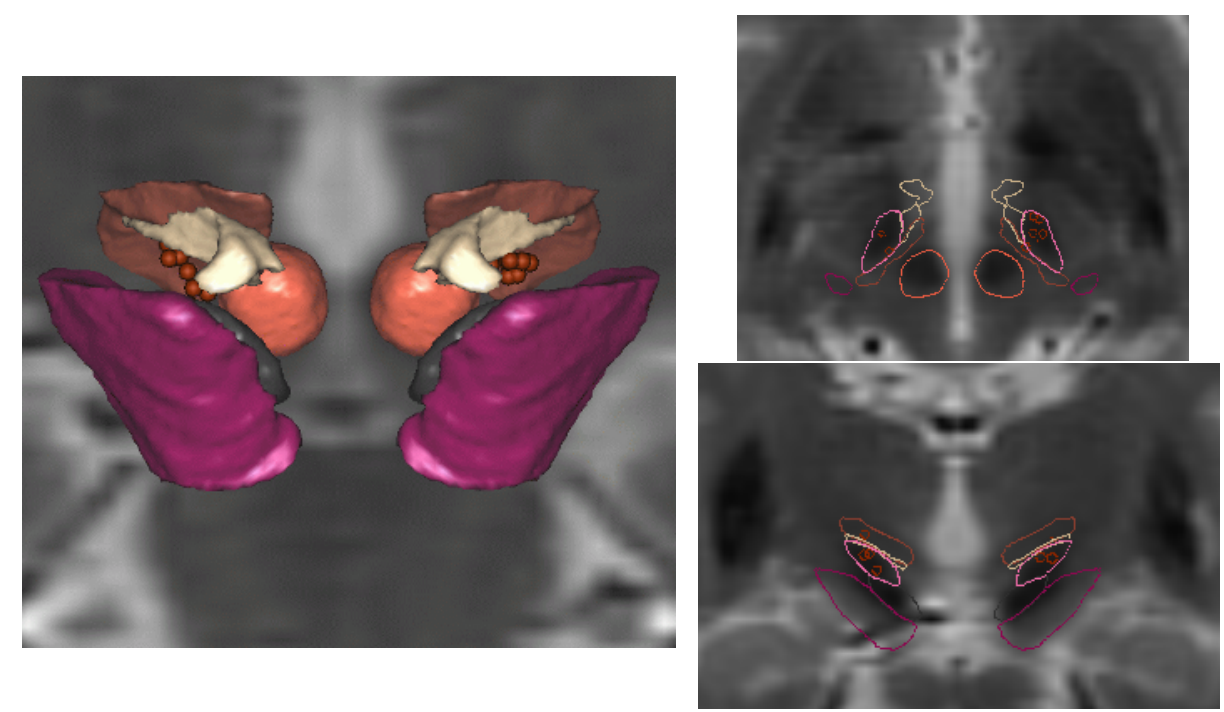

Fig. 4. Backprojection of the definitive therapeutic contacts in the atlas space. Left: STN surroundings - Cerebral Peduncle, Red Nucleus, Substancia Nigra, Zona Incerta, Forel Field II - the STN has been suppressed to allow visualization of the contacts (spheres); right: projection of the contacts onto the atlas T2 MR image (top: axial slice; bottom: coronal slice). Note that not all contacts are visible on these slices, as some contacts are located more dorsally or posteriorly.

be able to fuse this data with the atlas structures, the stereotactic frame was extracted from the T1 pre-operative MRI, then registered with a template of the frame to get the stereotactic referential. The stereotactic coordinates of 
the microelectrode recordings were converted to MRI coordinates, then each recording was encoded and displayed as a sphere, which size represents the intensity of the recorded signal, and which color represents the structure as identified by the electrophysiologist. For the six patients, 162 micro-electrode recordings were identified as STN by the electrophysiologist. Among them, 132 $(81.5 \%)$ were also inside the STN surface, obtained through automatic atlas deformation onto patient's T1-weighted pre-operative MR image. The remaining STN recordings were all located at less than $1 \mathrm{~mm}$ of the STN surface.

\section{Anatomical position of therapeutic contacts in Parkinsonian patients} treated by DBS Identification of the contacts on post-operative MR images was conducted following 2 steps: 1) MR images were reformatted along the electrodes trajectories; 2) the 3D atlas was deformed onto the post-operative MR images. This provided the anatomical position of the 4 contacts, including the therapeutic one. Fig. 4 presents all the therapeutic contacts backprojected into the 3D atlas space. For the 10 patients (meaning 20 therapeutic contacts), 14 contacts were located in the STN, 2 at the STH border, 2 in ZI (Zona Incerta) and 2 in $\mathrm{H} 2$ (Forel Field II).

\section{Discussion}

The average images illustrate that the atlas normalization is superior to the ACPC normalization, as the resulting mean images are much less blurry. Moreover, particular features are only clearly visible in the atlas-based normalized images (such as the ventral part of the putamen).

Concerning the comparison between atlas-based STN and STN revealed by electrophysiological recordings, it should be pointed out that the microelectrode recordings coordinates were given by the electrophysiologist as the distance between the electrode and the target. This distance was given by the microdrive with a variable initial offset of at most $1 \mathrm{~mm}$. This offset could explain some of the mismatches between electrophysiological data and atlas STN surfaces.

Semi-automatic registration based on the SW atlas [8] led, for 10 patients (20 electrodes) to 15 contacts located in the STN, 2 at the STN border, 1 in ZI, 1 in $\mathrm{H} 2$ and 1 in the MRF. The results obtained with the new $3 \mathrm{D}$ atlas automatically deformed onto patients post-operative MR images were very similar (see section 3.2), suggesting that the automatic 3D atlas MRI registration technique is reliable. The observed difference is most likely to be due to differences in the drawing of the two atlases (in particular for ZI and H2). Moreover, the SW atlas is a printed histological atlas in which the number of sections is low (20 sections in the frontal series) and the spacing between adjacent sections very variable $(1-4 \mathrm{~mm})$. In the new $3 \mathrm{D}$ atlas there are 160 sections for a 0.35 $\mathrm{mm}$ spacing. This means that in 8 a section corresponding to the position of a contact had to be registered with the nearest section in the SW atlas, which implies approximations. 
This study demonstrated a very good correlation between different methods for identifying the STN with an histological and deformable 3D atlas in different patients brains. In particular, comparison with STN revealed by microelectrode recordings led to good correspondance scores. Therefore it is concluded that this technique is reliable and that the current atlas/MRI coregistration could be used as a standard method of deep brain structures mapping. The method is currently validated through a large retrospective study of Parkinsonian patients treated by DBS.

\section{References}

1. Benabid, A.L., Pollak, P., Louveau, A., Henry, S., De Rougemont, J.: Combined (thalamotomy and stimulation) stereotactic surgery of the vim thalamic nucleus for bilateral parkinson disease. Appl. Neurophysiol. 50 (1987) 344-346

2. Limousin, P., Pollak, P., Benazzouz, A., Hoffmann, D., Broussolle, E., Perret, J.E., Benabid, A.L.: Bilateral subthalamic nucleus stimulation for severe parkinson's disease. Mov. Disord. 10 (1995) 672-674

3. Mallet, L., Mesnage, V., Houeto, J.L., Pelissolo, A., Yelnik, J., Behar, C., Gargiulo, M., Welter, M.L., Bonnet, A.M., Pillon, B., Cornu, P., Dormont, D., Pidoux, B., Allilaire, J.F., Agid, Y.: Compulsions, parkinson's disease, and stimulation. Lancet 360 (2002) 1302-1304

4. Bejjani, B.P., Dormont, D., Pidoux, B., Yelnik, J., Damier, P., Arnulf, I., Bonnet, A.M., Marsault, C., Agid, Y., Philippon, J., Cornu, P.: Bilateral subthalamic stimulation for parkinson's disease by using three-dimensional stereotactic magnetic resonance imaging and electrophysiological guidance. J. Neurosurg. 92 (2000) $615-625$

5. Mai, J.K., Assheuer, J., Paxinos, G.: Atlas of the human brain. Academic Press, San Diego (1997)

6. Morel, A., Magnin, M., Jeanmonod, D.: Multiarchitectonic and stereotactic atlas of the human thalamus. J. Comp. Neurol. 387 (1997) 588-630

7. Schaltenbrand, G., Wahren, W.: Atlas for stereotaxy of the human brain. Georg Thieme Verlag, Stuttgart (1977)

8. Yelnik, J., Damier, P., Demeret, S., Gervais, D., Bardinet, E., Bejjani, B.P., Franois, C., Houeto, J.L., Arnulf, I., Dormont, D., Galanaud, D., Pidoux, B., Cornu, P., Agid, Y.: Localization of stimulating electrodes in parkinsonian patients by using a three-dimensional atlas-magnetic resonance imaging coregistration method. J. Neurosurg. 99 (2003) 83-93

9. Niemann, K., Mennicken, V.R., Jeanmonod, D., Morel, A.: The morel stereotactic atlas of the human thalamus: atlas-to-mr registration of internally consistent canonical model. Neuroimage 12 (2000) 601-616

10. St-Jean, P., Sadikot, A., Collins, D., Clonda, D., Kasrai, R., Evans, A., Peters, T.: Automated atlas integration and interactive 3-dimensional visualization tools for planning and guidance of functional neurosurgery. IEEE Trans. Med. Imaging $\mathbf{1 7}$ (1998) 672-680

11. Nowinski, W., Belov, D., Benabid, A.: An algorithm for rapid calculation of a probabilistic functional atlas of subcortical structures from electrophysiological data collected during functional neurosurgery procedures. Neuroimage 18 (2003) 143155 
12. D’Haese, P., Cetinkaya, E.and Kao, C., Fitzpatrick, J., Konrad, P., Dawant, B.: Toward the creation of an electrophysiological atlas for the pre-operative planning and intra-operative guidance of deep brain stimulators (dbs) implantation. In: MICCAI 2004, Proceedings. Volume 3216 of LNCS., Springer (2004) 729-736

13. Duerden, E., Finnis, K., Peters, T., AF, S.: A method for analysis of electrophysiological responses obtained from the motor fibers of the human internal capsule. In: MICCAI 2003, Proceedings. Volume 2878 of LNCS., Springer (2003) 50-57

14. Finnis, K., Starreveld, Y., Parrent, A., Sadikot, A., Peters, T.: Three-dimensional database of subcortical electrophysiology for image-guided stereotactic functional neurosurgery. IEEE Trans. Med. Imaging 22 (2003) 93-104

15. Bardinet, E., Ourselin, S., Dormont, D., Malandain, G., Tandé, D., Parain, K., Ayache, N., Yelnik, J.: Co-registration of histological, optical and MR data of the human brain. In: MICCAI 2002, Proceedings. Volume 2488 of LNCS., Springer (2002) 548-555 